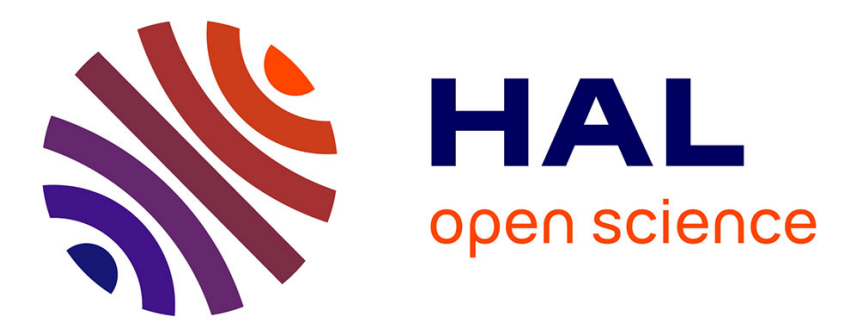

\title{
Light-Driven Molecular Motors Boost the Selective Transport of Alkali Metal Ions through Phospholipid Bilayers
}

\author{
Wen-Zhi Wang, Li-Bo Huang, Shao-Ping Zheng, Emilie Moulin, Odile Gavat, \\ Mihail Barboiu, Nicolas Giuseppone
}

\section{To cite this version:}

Wen-Zhi Wang, Li-Bo Huang, Shao-Ping Zheng, Emilie Moulin, Odile Gavat, et al.. Light-Driven Molecular Motors Boost the Selective Transport of Alkali Metal Ions through Phospholipid Bilayers. Journal of the American Chemical Society, 2021, 143 (38), pp.15653-15660. 10.1021/jacs.1c05750 . hal-03410237

\section{HAL Id: hal-03410237 \\ https://hal.science/hal-03410237}

Submitted on 9 Nov 2021

HAL is a multi-disciplinary open access archive for the deposit and dissemination of scientific research documents, whether they are published or not. The documents may come from teaching and research institutions in France or abroad, or from public or private research centers.
L'archive ouverte pluridisciplinaire HAL, est destinée au dépôt et à la diffusion de documents scientifiques de niveau recherche, publiés ou non, émanant des établissements d'enseignement et de recherche français ou étrangers, des laboratoires publics ou privés. 


\title{
Light-driven molecular motors boost the selective transport of alkali metal ions through phospholipid bilayers
}

\author{
Wen-Zhi Wang, ${ }^{[\mathrm{a}]}$ Li-Bo Huang, ${ }^{[\mathrm{b}]}$ Shao-Ping Zheng, ${ }^{[\mathrm{b}]}$ Emilie Moulin, ${ }^{[\mathrm{a}]}$ Odile Gavat, ${ }^{[\mathrm{a}]}$ Mihail Bar- \\ boiu, ${ }^{*[\mathrm{~b}]}$ Nicolas Giuseppone*[a] \\ [a] SAMS Research Group, Université de Strasbourg, CNRS, Institut Charles Sadron UPR22, 67000 Strasbourg, France \\ [b] Adaptive Supramolecular Nanosystems Group, Institut Européen des Membranes (IEM), University of Montpellier, 300 \\ avenue du Professeur Emile Jeanbrau, 34000 Montpellier, France
}

\begin{abstract}
A hydrophobic light-driven rotary motor is functionalized with two 18-crown-6 macrocycles and incorporated into phospholipid bilayers. In the presence of this molecular construct, fluorescence assays and patch clamp experiments show the formation of selective alkali ion channels through the membrane. Further, they reveal a strongly accelerated ion transport mechanism under light irradiation. This increase of the fractional ion transport activity (up to 400\%) is attributed to the out-of-equilibrium actuation dynamics of the light-driven rotary motors that helps overcoming the activation energy necessary to achieve translocation of alkali ions between macrocycles along the artificial channels.
\end{abstract}

\section{INTRODUCTION}

The selective transport of ions across lipid bilayers is a key process to ensure integrity of living cells and to provide them with advanced functionalities. ${ }^{1-3}$ If simple biomolecular carriers process by a passive mode of transport, more sophisticated protein channels can involve channel-based regulation mechanisms (or even active transport) by using external effectors (or a source of energy). Beyond natural ion channels, synthetic artificial pores, with a few of them including shuttling molecular machines, ${ }^{4-7}$ have demonstrated remarkable applicative interests for sensing, separation, and delivery processes. ${ }^{8-10}$ In particular, light-controlled molecular systems offer the opportunity to govern, under temporal and spatial control, a large number of ionic translocation processes through bilayer membranes. ${ }^{11,12}$ Light-gated photochromic ligands have been designed to change the pore diameter or the ion-dipole action of protein ${ }^{13}$ and artificial $^{14}$ channels. Light-regulated carriers ${ }^{15}$ or channels ${ }^{16-18}$ with individual ion-conduction states in bilayer membranes can be optically switched between different states via photo-isomerization of their former components. The operational range of photo-switches can also be used to create local molecular motions inside the pores of mesoporous silica and to facilitate the release of pore-confined molecules. ${ }^{19}$ Interestingly, following different approaches and objectives, hydrophobic high-frequency $(\mathrm{MHz})$ light-driven rotary motors were recently integrated in the membrane of living cells, leading to their death by drilling of the membrane and subsequent leakage of their cytoplasm content. ${ }^{20}$ The rotation of such motors was also demonstrated in the phospholipid bilayers of artificial liposomes or within other amphiphilic structures leading also to their drilling or to their morphological change at mesoscale..$^{21,22}$ However, to the best of our knowledge, the implementation of light-driven molecular motors ${ }^{23-25}$ has not been yet envisioned for regulating selective ion-transport processes across lipid membranes. Now we report that light-driven rotary motors conjugated with crown ethers can self-assemble in ion channels within phospholipid bilayers, and further boost their selective ion transport activity when actuating their motor part under UV irradiation.

\section{RESULTS AND DISCUSSION}

This work started with the synthesis of molecular motor A (Scheme 1), which integrates a urea motif to favor its supramolecular self-assembly by hydrogen bonds within the phospholipid bilayer. Besides, it includes an 18-crown-6 ether as macrocyclic unit, which is well known to perfectly accommodate potassium cations in its center and to form sandwich-type complexes with rubidium cations. ${ }^{26,27}$ Motor A was prepared in three steps from molecular motor $\mathbf{1}^{28,29}$ and crown ether derivative 4, as shown in Scheme 1. To access derivative 4 (Scheme 1b), compound $\mathbf{5}$ was first synthesized from commercially available 4-nitrocatechol and pentaethylene glycol di(p-toluenesulfonate) in the presence of templating potassium cations to favor macrocyclisation. Further reduction of the nitro group with palladium on charcoal and hydrazine afford compound $\mathbf{6}$, followed by condensation of the resulting amine with 2-chloroethyl isocyanate, afforded crown ether macrocycle 7 in reasonable yields. Subsequent nucleophilic substitution with sodium azide, and reduction of the azide to the amine using the Staudinger reaction, afforded compound $\mathbf{4}$ in good yields. On the other hand (Scheme 1a), free phenol groups of already reported molecular motor $\mathbf{1}^{28,29}$ were protected by nucleophilic substitutions with bromoethane. Subsequent saponification of the two ethyl esters using sodium hydroxide in a mixture of THF and methanol afforded molecular motor 3. Finally, compound $\mathbf{A}$ was obtained by an amide coupling reaction between amine 4 and bis-acid 3 using 1-Ethyl-3-(3-dimethylaminopropyl)carbodiimide (EDC) and HOBt as reagents. This key compound was purified by preparative reverse-phase high-performance liquid chromatography (HPLC). 
a)
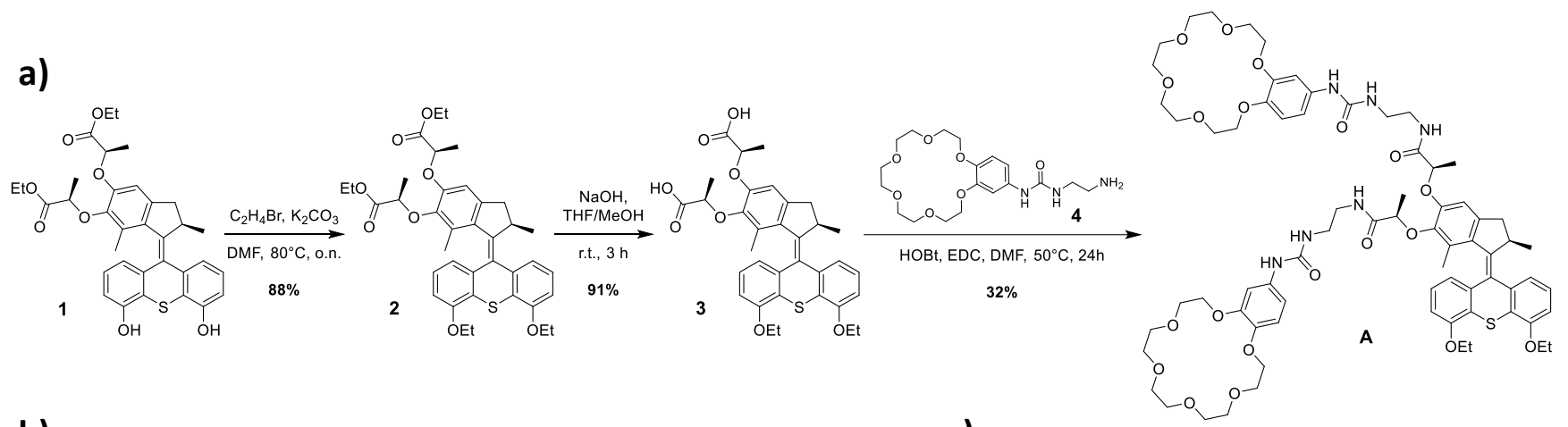

b)
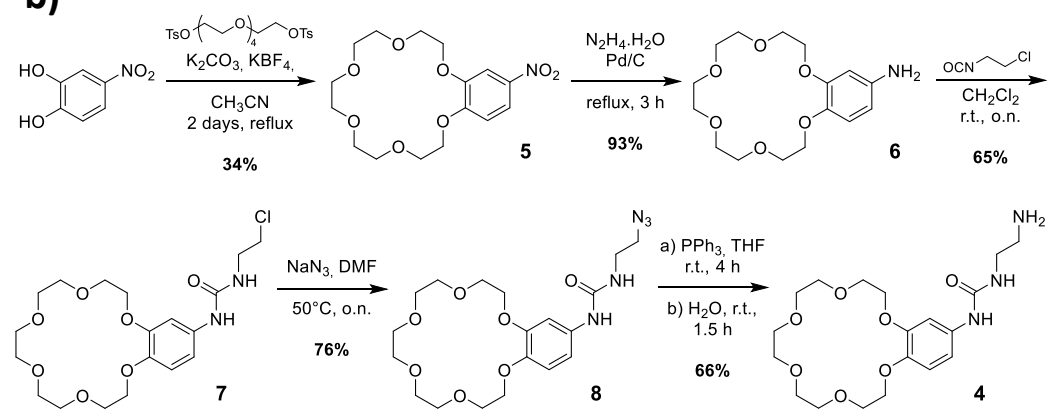

c)

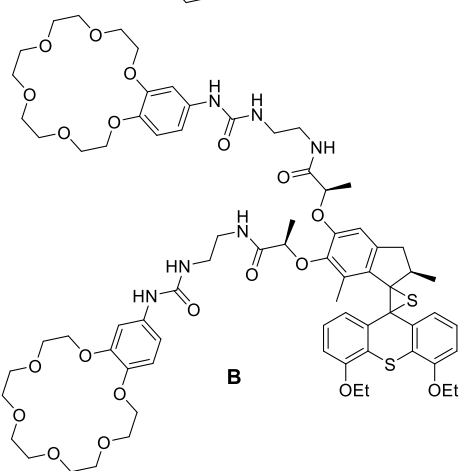

Scheme 1. Synthetic routes for a) molecular motor $A$ and b) crown ether derivative 4, c) chemical structure of episulfide $B$.

In addition, to better probe the influence of the rotation of molecular motor $\mathbf{A}$ on the ion transport properties upon light irradiation, we also synthesized episulfide $\mathbf{B}$ as a "control" molecule in which the central photoactuating double bond of the motor is replaced by an episulfide moiety, thereby precluding any rotation upon light irradiation (Scheme 1c). Starting from episulfide $\mathbf{9},{ }^{29}$ compound $\mathbf{B}$ was synthesized in three steps according to the same synthetic pathway as for compound $\mathbf{A}$ (Scheme S2). The expected chemical structure and purity of all synthetic products were confirmed by NMR spectroscopy and ESI analysis (see SI for detailed synthetic protocols and characterizations).

The transport of alkali metal ions through lipid bilayers in the presence of molecular motor A was monitored by a HPTS fluorescence assay on Large Unilamellar Vesicles (LUVs) of phosphatidylcholine. ${ }^{30}$ In this assay (Figure 1a), the fluorescent probe HPTS (8-hydroxypyrene-1,3,6-trisulfonic acid, pKa = 7.2 ), which in its protonated and deprotonated states exhibits two different absorption wavelengths (450 and $405 \mathrm{~nm}$ respectively), is used as a $\mathrm{pH}$ probe inside the LUVs in order to indirectly measure the transport of alkali cations $\mathrm{M}^{+}$. After introduction of $\mathbf{A}$ within the bilayer, the addition of sodium hydroxide solution to the extravesicular medium creates a pH gradient of approximately one unit compared to the intravesicular medium. Because of the presence of $\mathbf{A}$, this gradient can then be compensated by cation/proton antiport or by metal-cation/hydroxide co-transport. ${ }^{30,31}$ By monitoring the relative fluorescence intensity $\left(\mathrm{I}_{450} / \mathrm{I}_{405}\right)$ of the HPTS probe, one can measure an increase of the intravesicular $\mathrm{pH}$ which reflects the alkali cation transport across the membrane.

Preliminary to this experiment, we probed the solubility of molecular motor A into Egg yolk L- $\alpha$-phosphatidylcholine (EYPC) LUVs by UV-Vis spectroscopy. The linearity of the Beer-Lambert law was verified in the presence of the LUVs, and we determined an extinction coefficient $\varepsilon=1.4410^{4} \mathrm{~L} . \mathrm{mol}$
${ }^{1} . \mathrm{cm}^{-1}$ in the conditions of the assay (see SI section S4 and Figures $\mathrm{S} 2$ for details). Figure $1 \mathrm{~b}$ shows that for a same concentration of $\mathbf{A}(33 \mu \mathrm{M})$ in a $\mathrm{KCl} /$ Phosphate-buffered saline (PBS) solution, the absorption spectrum of the motor increases its intensity by more than 4 times in the presence of LUVs, indicating that, as expected, most of the hydrophobic motor is present within the phospholipid bilayers during the HPTS fluorescence assay. In addition, we measured by dynamic light scattering (DLS) the size of the vesicles, which show similar diameters of $100 \mathrm{~nm}$ with and without molecular motors (Figure S4).

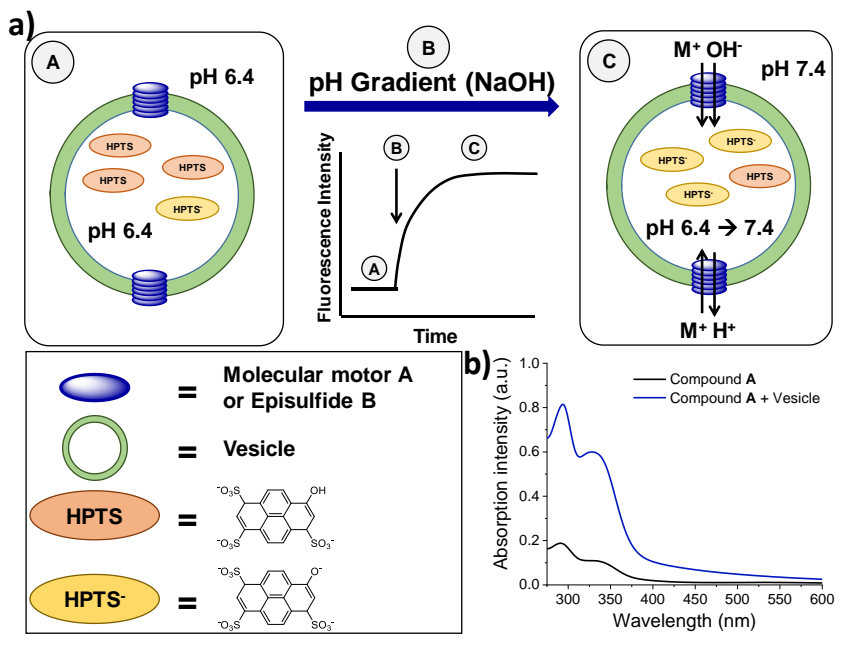

Figure 1. a) Schematic representation of the HPTS fluorescence assay implemented here to study the transport of alkali metal cations through phospholipid bilayer membranes incorporating molecular motor A. b) UV-Vis spectra of motor $\mathbf{A}(\mathrm{c}=33 \mu \mathrm{M})$ in a $\mathrm{KCl}$ / PBS medium, in the presence (blue) or the absence (black) of LUVs (see SI, section S4 for details).

We also probed as a control experiment that the typical UV irradiation used in this entire study $\left(365 \mathrm{~nm}, 7.5 \mathrm{~mW} . \mathrm{cm}^{-2}\right.$, for 
$10 \mathrm{~min}$ ) of the vesicles loaded with motors does not alter their integrity neither their size (see SI section 5). With this preliminary information, we evaluated the transport activities of $\mathrm{Na}^{+}$, $\mathrm{K}^{+}$and $\mathrm{Rb}^{+}$cations with increasing concentrations of compound A within bilayer membranes and in the presence of continuous, partial and no UV light irradiation at $365 \mathrm{~nm}$. EYPC LUVs (100 nm diameter) were filled with HPTS and $100 \mathrm{mM} \mathrm{NaCl}$ in a phosphate buffer solution (10 mM, pH 6.4). The liposomes were then suspended in an external phosphate buffer solution (10 mM, pH 6.4) containing $100 \mathrm{mM}$ of either $\mathrm{NaCl}, \mathrm{KCl}$ or $\mathrm{RbCl}$. After addition of $20 \mu \mathrm{L}$ of a stock solution of molecular motor $\mathbf{A}$ in DMSO (2-10 mM), the effective concentration of $\mathbf{A}$ was in the range of $20-100 \mu \mathrm{M}$. A pH gradient was then generated upon external addition of $\mathrm{NaOH}$, and the internal $\mathrm{pH}$ change (i.e. inside the liposome) was monitored by the change in fluorescence of HPTS. For each batch of experiments, control experiments were recorded in the absence of $\mathbf{A}$ (with and without UV irradiation), to evaluate if ionic diffusion through the lipids bilayer might be affected by UV irradiation, that is by rotation of the molecular motor (Figure 2).

In all the experiments performed, we confirmed the negligible transport of alkali ions in the absence of $\mathbf{A}$, independently of the UV irradiation of the vesicles. Importantly, without UV irradiation and in the presence of $\mathbf{A}$, a strong increase of transport activity was measured compared to the control experiment without A (Figures 2a-c, blue and black curves, respectively). Variations of the fractional ion transport profiles were also shown to depend on the nature of the alkali cation present on the outer shell of the vesicle (see for instance at a concentration of $80 \mu \mathrm{M}$ in $\mathbf{A}$, the effect on the transport of $\mathrm{NaCl}$ (Figure 2a),
$\mathrm{KCl}$ (Figure 2b) and $\mathrm{RbCl}$ (Figure 2c), with the following relative rates of transport $\left.\mathrm{Rb}^{+}>\mathrm{K}^{+}>\mathrm{Na}^{+}\right)$. In addition, when evaluating the transport activity in the dark with increasing concentration of A (Figure 2d-f, blue bars), one can evidence that compound $\mathbf{A}$ forms aggregated ion-channels towards cations, and Hill coefficients were determined for these three metal ions (see SI section S2.2 and Figure S1). The Hill coefficients are $n>1$ for all cations, meaning that more than one active molecule of $\mathrm{A}$ is used to generate channels with a high degree of cooperativity for the selective transport of cations. The use of benzo-18crown- 6 as a cationic recognition site in the bilayer membrane induces adaptive transport behaviors for compound $\mathbf{A}$ with a selectivity for $\mathrm{K}^{+}$at low and medium concentrations $(0-40 \mu \mathrm{M})$, which then evolves for $\mathrm{Rb}^{+}$cations at high concentrations $(60-$ $100 \mu \mathrm{M})$. Although the exact translocation mechanism of the cations remains difficult to describe precisely at this stage, we know from previous studies ${ }^{26}$ that macrocyclic compounds recognize the fittest cations via an equatorial binding, whereas a selective sandwich macrocyclic recognition occurs to completely surround the bigger cations. This has been systematically rationalized for ion-specific chromatographic, ${ }^{32}$ membrane $^{33}$ and sensing ${ }^{34,35}$ polymeric materials. Such a behavior is also reminiscent with the spatial positioning of the carbonyl moieties, perfectly replacing the hydration sphere of $\mathrm{K}^{+}$cations within active gate of $\mathrm{KcsA} \mathrm{K}^{+}$natural channel. ${ }^{10}$ In the present case, it is therefore highly possible that the cations translocate through self-assembled macrocyclic cation-binding aggregates involving proximal macrocycles that may create dynamic pores within the membrane environment.
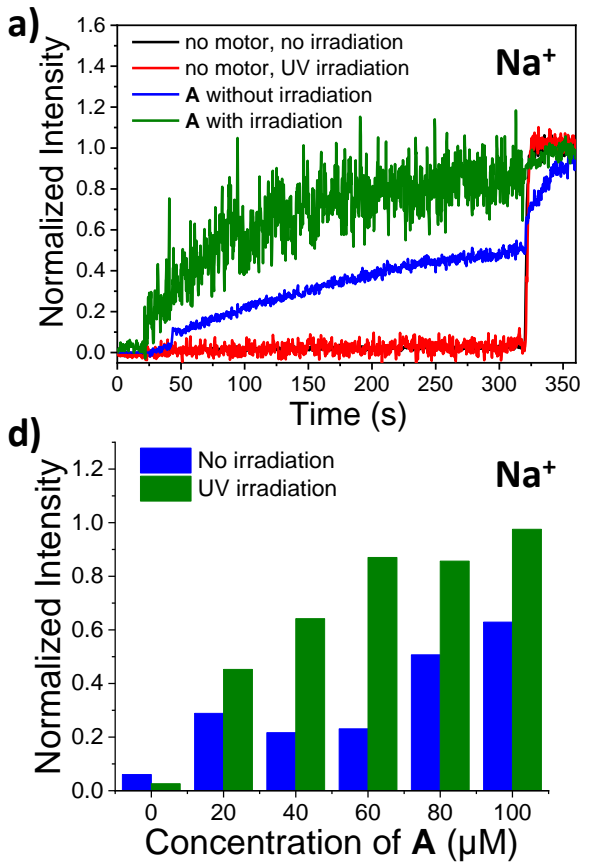
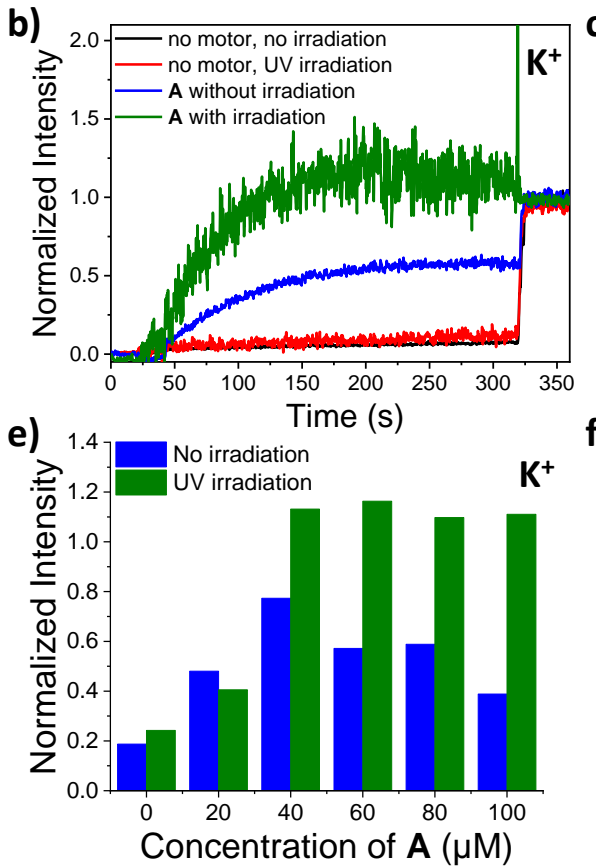
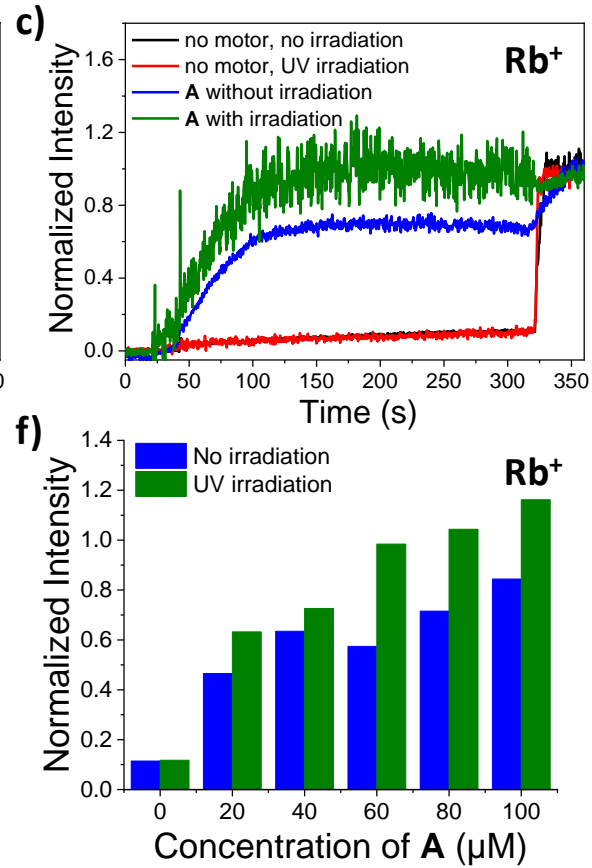

Figure 2. a-c) Evolution of the fractional cation transport activity as a function of time for a) $\left.\mathrm{Na}^{+}, \mathbf{b}\right) \mathrm{K}^{+}$and $\mathbf{c}$ ) $\mathrm{Rb}^{+}$cations in the absence of molecular motor A and without (black) or with (red) UV irradiation ( $365 \mathrm{~nm}, 7.5 \mathrm{~mW} . \mathrm{cm}^{-2}$, for $330 \mathrm{~s}$ ); and in the presence of a $80 \mu \mathrm{M}$ of molecular motor A and without (blue) or with (green) UV irradiation (365 nm for $330 \mathrm{~s}$ ); d-f) Evolution of the fractional cation transport activity as a function of the concentration of molecular motor $\mathbf{A}$ in the bilayer membrane at $300 \mathrm{~s}$ for d) $\mathrm{Na}^{+}$, e) $\mathrm{K}^{+}$and f) $\mathrm{Rb}^{+}$without (blue) and with (green) UV irradiation. Internal composition of LUV is $100 \mathrm{mM} \mathrm{NaCl}, 10 \mathrm{mM}$ phosphate buffer at pH 6.4 and $\mathrm{HPTS} 10 \mu \mathrm{M}$. External compositions are $100 \mathrm{mM}$ of $\mathbf{a , d}) \mathrm{NaCl}, \mathbf{b}, \mathbf{e}) \mathrm{KCl}$ or $\mathbf{c , f}) \mathrm{RbCl}$ in $10 \mathrm{mM}$ phosphate buffer at pH 6.4. 


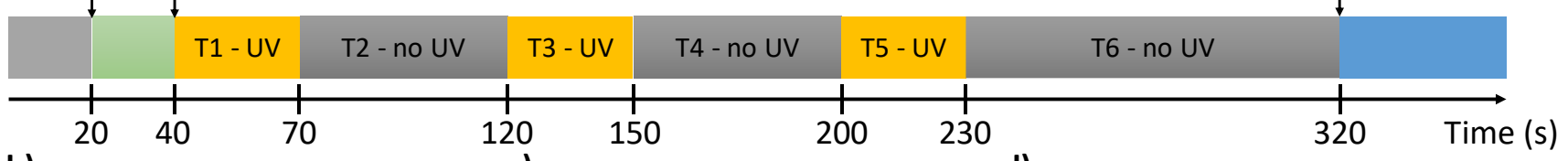
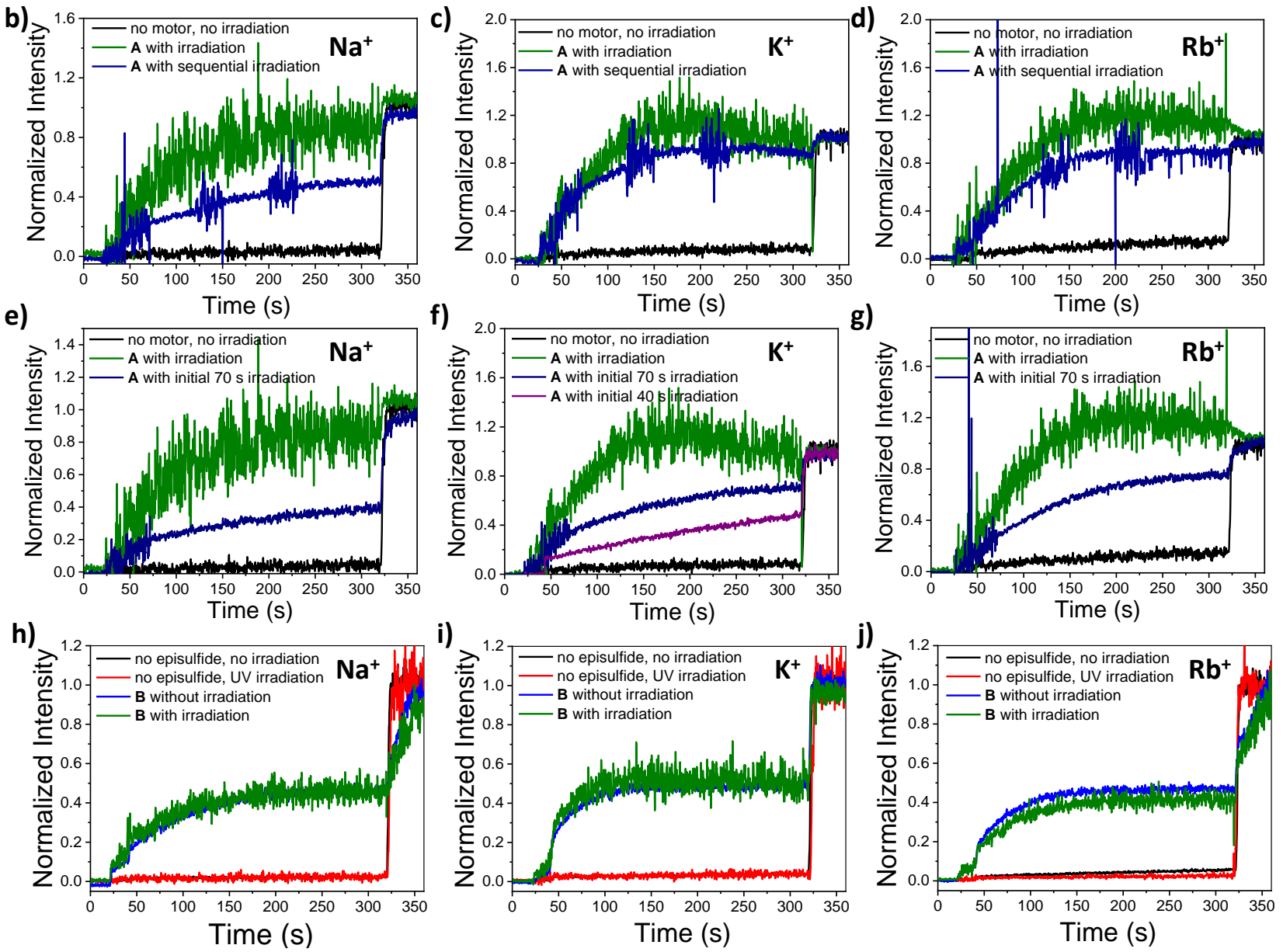

Figure 3. a) Sequential UV irradiation protocol over $360 \mathrm{~s}$ for ion transport experiments: at $20 \mathrm{~s}$, addition of compound A; no UV irradiation from 20 to $40 \mathrm{~s}$; UV irradiation at $365 \mathrm{~nm}$ from 40 to $70 \mathrm{~s}$ (T1), 120 to $150 \mathrm{~s} \mathrm{(T3),} \mathrm{and} 200$ to $230 \mathrm{~s}$ (T5); no UV irradiation from 70 to $120 \mathrm{~s}$ (T2), 150 to $200 \mathrm{~s}$ (T4), and 230 to $320 \mathrm{~s}$ (T6); b-d) Evolution of the fractional ion transport activity $\left(\mathrm{C}_{\mathrm{A}}=40 \mu \mathrm{M}\right)$ as a function of time for b) $\mathrm{Na}^{+}$, c) $\mathrm{K}^{+}$and d) $\mathrm{Rb}^{+}$in the absence of UV light (black), upon continuous UV irradiation (red) and according to the sequential UV irradiation process (blue); e-g) Evolution of the fractional ion transport activity $\left(\mathrm{C}_{\mathrm{A}}=40 \mu \mathrm{M}\right)$ as a function of time for e) $\left.\mathrm{Na}^{+}, \mathbf{f}\right) \mathrm{K}^{+}$and g) $\mathrm{Rb}^{+}$in the absence of UV light (black), upon continuous UV irradiation (red), according to an initial 70-second UV irradiation process (blue) and according to the initial 40-second UV irradiation process (purple); h-i) Evolution of the fractional ion transport activity as a function of time for $\mathbf{h}) \mathrm{Na}^{+}$, i) $\mathrm{K}^{+}$and j) $\mathrm{Rb}^{+}$in the absence of UV light and of episulfide (black), in the presence of UV light but without episulfide (red), in the presence of episulfide $\mathbf{B}$ but without UV irradiation (blue), and in the presence of episulfide $\mathbf{B}$ along with UV irradiation (green), with $\mathrm{C}_{\mathrm{B}}=100 \mu \mathrm{M}$.

We then probed with the same HPTS assay the effect of UV irradiation, and therefore indirectly the effect of molecular motor rotation, on the transport activity of $\mathrm{Na}^{+}, \mathrm{K}^{+}$, and $\mathrm{Rb}^{+}$(Figure $2 \mathrm{a}-\mathrm{c}$, green curves). Satisfyingly, experiments under UV light revealed a very different picture from the results obtained in the dark. Fractional activity increases in all cases in the presence of UV light, and with the strongest improvement of $400 \%$ observed for $\mathrm{Na}^{+}$in the presence of $\mathbf{A}$ at a concentration of $60 \mu \mathrm{M}$. Because, in all cases, the fractional activity is at least one order of magnitude higher than the one observed under UV irradiation but in the absence of $\mathbf{A}$, one can postulate that this increase is the result of a mechanically activated transport mechanism. Selectivity of transport is also observed under light irradiation, with for instance $\mathrm{S}_{\mathrm{K}+\mathrm{Na}+}=1.8$ at a concentration of $40 \mu \mathrm{M}$ in $\mathbf{A}$. This preference for $\mathrm{K}^{+}$cations decreases in favor of $\mathrm{Rb}^{+}$cations at higher concentrations and $\mathrm{S}_{\mathrm{Rb}+/ \mathrm{K}+}=2$, following the same trend already observed without light irradiation. Overall, the general trend observed in these experiments correspond to a strong acceleration of the transport process in the presence of light irradiation, while preserving selectivity aspects between the different ions as a function of the concentration in $\mathbf{A}$.

We also studied if this intriguing light-activated transport is the result of an irreversible triggered process, or of a reversible process requiring a continuous irradiation to function. To probe this behavior, we performed sequential irradiation experiments (see protocol of irradiation described in Figure 3a) with the 
same HPTS assay and with a $40 \mu \mathrm{M}$ concentration in $\mathbf{A}$. For the 3 metal ions, the alternation of UV irradiation and of dark periods shows that the overall intensity of the transport is reduced by the presence of light-off periods (Figure 3b-d). One can even notice that by just lighting the system at the beginning of the transport for only $70 \mathrm{~s}$ (Figures 3e-g) or 40s (Figure 3f), the accelerated transport rate drops immediately when switching off the light, and reaches a transport regime which is comparable with the "no light" reference experiment (Figure 2a-c). In addition, both continuous and sequential UV experiments lead to a final saturation transport behavior, due to the decrease of the $\mathrm{M}^{+}$ concentration gradient between the intra- and extra-vesicular media, and the increase of the $\mathrm{M}^{+}$concentration in the intravesicular medium. Therefore, the main driving force that generates the cation influx is the transmembrane concentration potential, but this is supplemented by a specific UV activation of molecular motors. In addition, the boosted transport of the metal ions under UV irradiation is a reversible process which immediately stops when the light is switched off.

To clarify whether the mechanism involved in the acceleration of the ion transport was related to a configurational or conformational switch of molecule $\mathbf{A}$, or rather to its out-of-equilibrium rotational motion, we performed a series of 4 control experiments $(i-i v) . i)$ We first studied the influence of UV irradiation on control episulfide $\mathbf{B}$ (Scheme 1) in which the central photo-actuating double bond of the motor is replaced by an episulfide moiety, thereby precluding any rotation upon light irradiation. Figures 3h-j show the results for $\mathrm{Na}^{+}, \mathrm{K}^{+}$, and $\mathrm{Rb}^{+}$cations respectively, and unambiguously indicate that there is no enhancement of transport generated by locked motor $\mathbf{B}$, thereby strongly suggesting that the rotation motion of $\mathbf{A}$ is essential to boost the ion transport. ii) To verify this rotation hypothesis, we recorded UV and CD spectra of motor $\mathbf{A}$ in the precise conditions of the fluorescent assay (that is in the presence of the LUVs and $\mathrm{K}^{+}$ions). As shown in Figure S3, no change was observed after 5 or 10 minutes of UV irradiation, indicating the absence of another isomer of $\mathbf{A}$ in the system. In particular, the presence of the unstable helix form of $\mathbf{A}$ should be visible if its lifetime was long enough. This control experiment therefore shows that either the motor is not turning, or that it is turning too fast to detect the unstable helix (as it is the case for this type of second generation rotary motors with $\mathrm{MHz}$ frequencies due to a low activation energy of thermal helix inversion in solution). ${ }^{29,36}$ iii) To probe whether the rotation indeed takes place in the phospholipid membrane, we synthesized a dissymmetric motor $\mathbf{C}$, having in its bottom part a phenol group and a ethoxy group (instead of two ethoxy groups in $\mathbf{A}$ ). This structure allows to differentiate between the E/Z isomers by ${ }^{1} \mathrm{H}$ NMR, and even to separate them by preparative HPLC (see SI section S6). By isolating one isomer of $\mathbf{C}$ and by irradiating it in the LUVs and in the presence of $\mathrm{K}^{+}$ions, we proved the formation of the other configurational isomer resulting from $\mathrm{E} / \mathrm{Z}$ photoisomerization, and therefore the rotation of the motor (see Figure S5). iv) As a last control experiment, we performed the HPTS assay with variable intensities of UV light, showing that the rate of the transport increases with more powerful irradiations (with a saturation effect above $7.5 \mathrm{~mW} . \mathrm{cm}^{-2}$ ), thus strongly suggesting that the speed of the rotation is related to the transport efficiency (Figure 4a-b).

To understand the importance of the chemical structure of $\mathbf{A}$ in boosting the ion transport, we performed a second set of 3 control experiments (i-iii). i) First of all, we probed the importance of the macrocycle in channeling potassium ions by performing the fluorescent assay with motor 2 alone (which not contain crown ethers). No transport was observed both in the dark and under UV irradiation, which indicates in addition that no drilling of the phospholipid membrane by the motor takes place in these conditions (Figure S6). ii) Then, we performed the same experiment but with adding crown ether $4^{, 26}$ in addition of motor 2 (Figure 4c). In that case, a modest transport of $\mathrm{K}^{+}$ions was measured in the dark, and with no acceleration under UV irradiation, which indicates the necessity to link covalently the crown part and the motor part to benefit from its mechanical actuations. iii) We also performed an experiment with motor D containing 4 macrocycles (see SI section S8). No transport was observed in the dark or with light irradiation, possibly because of a too high steric hindrance of the molecular structure avoiding the formation of ion channels (Figure S7).
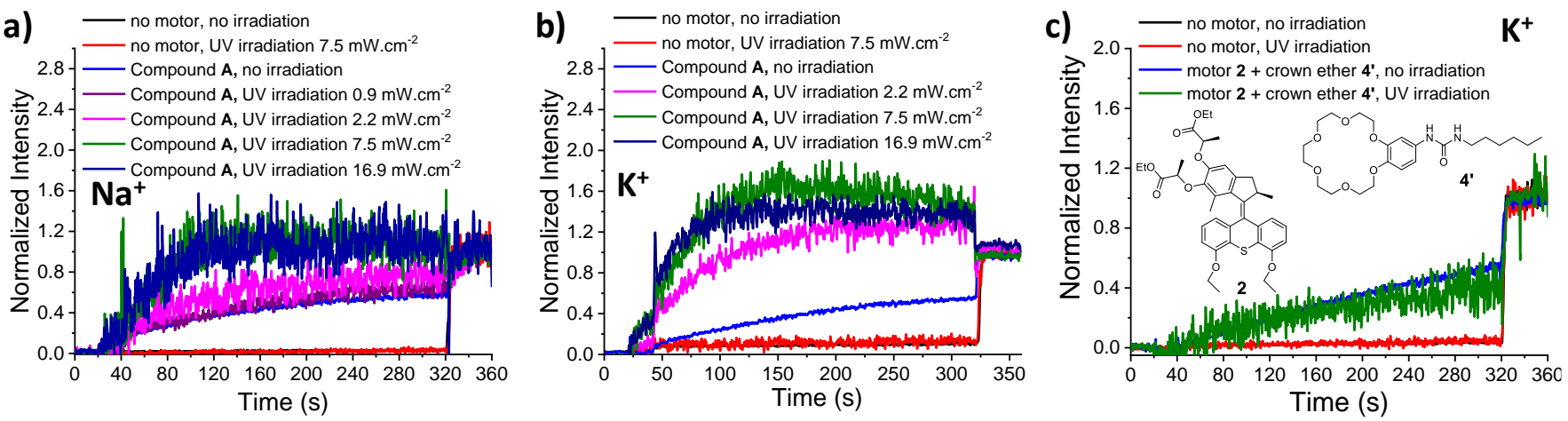

Figure 4. a-b) Evolution of the fractional ion transport activity $\left(\mathrm{C}_{\mathrm{A}}=40 \mu \mathrm{M}\right)$ as a function of time for a) $\mathrm{Na}^{+}$, and $\left.\mathbf{b}\right) \mathrm{K}^{+}$in the absence of UV light (blue), and for different UV irradiation intensities $0.9 \mathrm{~mW} . \mathrm{cm}^{-2}$ (purple), $2.2 \mathrm{~mW} . \mathrm{cm}^{-2}$ (pink), $7.5 \mathrm{~mW} . \mathrm{cm}^{-2}$ (green) and 16.9 $\mathrm{mW} . \mathrm{cm}^{-2}$ (dark blue), traces in black and red corresponds to measurements performed in the absence of motor and in the absence of UV light (black) or with an UV irradiation intensity of $7.5 \mathrm{~mW} . \mathrm{cm}^{-2}$ (red); c) Evolution of the fractional ion transport activity as a function of time for $\mathrm{K}^{+}$in the absence of UV light and of motor (black), in the presence of UV light but without motor (red), in the presence of motor 2 and crown ether 4' but without UV irradiation (blue), and in the presence of motor $\mathbf{2}$ and crown ether 4' along with UV irradiation (green), with $\mathrm{C}_{2}=\mathrm{C}_{4},=60 \mu \mathrm{M}$. 
Finally, to obtain supplementary evidences on the ion translocation mechanism through macrocyclic superstructures, we performed a set of conductance experiments with potassium cations. For that, we used the planar lipid bilayer clamp technique ${ }^{31}$ in a symmetrical $1 \mathrm{M} \mathrm{KCl}$ bath solution as electrolyte, with or without UV irradiation (see SI for detailed procedures). After addition of compound $\mathbf{A}$, the transport activity is rather slow to initiate, often requiring $20 \mathrm{~min}$ before detecting any onset of activity, which suggests that time is required to self-assemble the channel structure (Figure 5a). When active, at a concentration of $80 \mu \mathrm{M}$ in $\mathbf{A}$, transitions between closed and opened states are observed, describing long events (1-2 s) without UV irradiation. These events become shorter $(<0.5 \mathrm{~s})$ with UV irradiation, and with an average opened state conductance around 200 pS (multi-level conductance type).
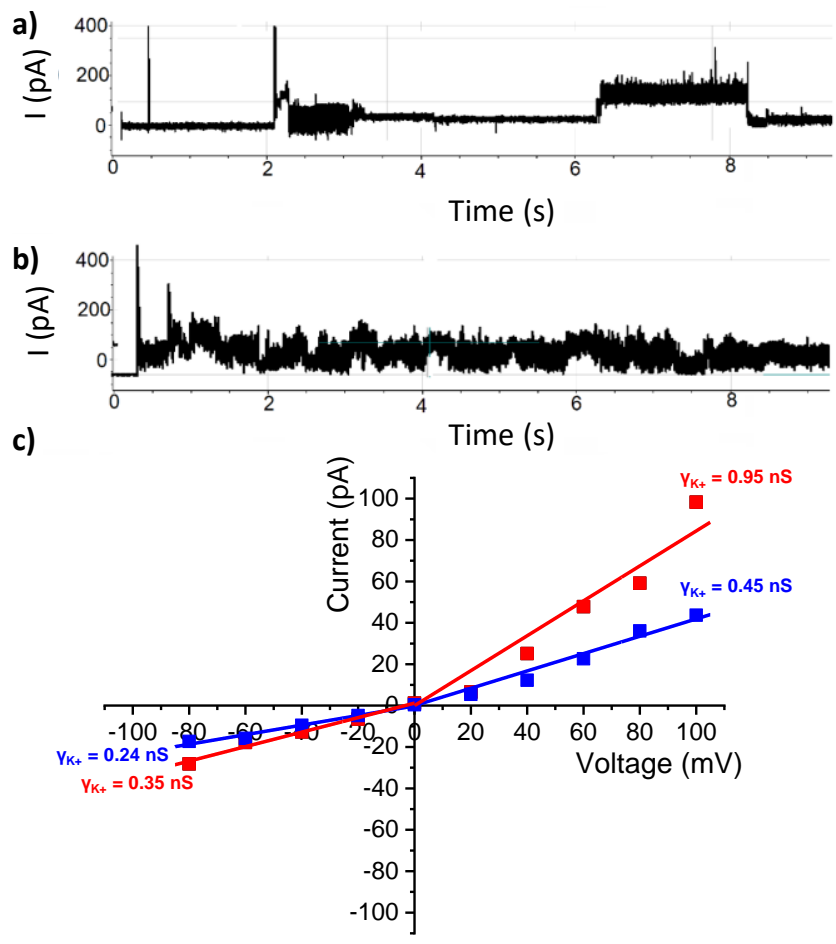

Figure 5. Typical evolution of the membrane activity measured by a single channel planar bilayer experiments involving motor $\mathbf{A}$ a) without UV and b) under UV irradiation (Cis/trans $1 \mathrm{M} \mathrm{KCl}$, diphytanoylphosphatidylcholine (diPhyPC), $80 \mu \mathrm{M} \mathrm{A},+100 \mathrm{mV}$ ). c) I-V plots for channels made of compound $\mathbf{A}$ recorded with UV (red) and without UV (blue) irradiation.

The activity without UV irradiation (Figure 5a) suggests the formation of unstable single channels with an erratic activity produced as periodic and occasional bursts and possibly arising from unstable pores. ${ }^{37}$ In the present case, evaluation of the overall conductance of the open states is more likely related to dynamic exchanges between channel components rather than copies of a single channel. ${ }^{38}$ These open states events are highly amplified under UV irradiation (Figure 5b), and therefore supposing by the mechanical rotation of molecular motor $\mathbf{A}$. Their orderly flow toward one direction creates a dynamic charge distribution along the channel, which can certainly be stimulated if a strong electric field is applied. Thus, this multiple conductance behavior is intrinsically related to a type of 'supramolecular polymorphism' while the cation translocation is related to the dynamics of the crown-ether aggregates within bilayers. In addition, despite the presence of local signal variability due to dynamic behaviors of the lipid bilayers under combined UV irradiation/mechanical motor motions, the presence of open and close states under light irradiation in the patch clamp experiment - together with the ion selectivity revealed by the HPTS assay as well as the absence of transport with motor 2 alone -, strongly discard the possibility of a transport mechanism resulting from the drilling of the membrane $.^{20,21} \mathrm{I}-\mathrm{V}$ plots in Figure $4 \mathrm{~b}$ also show a linear variation (i.e., ohmic behavior) at low potential, while at higher potential they become asymmetric, suggesting that channels of $\mathbf{A}$ can be triggered at high positive voltage. The ionic conductance values $\left(\gamma_{\mathrm{K}+}\right)$ are $0.45 \pm 0.04 \mathrm{nS}$ (without UV irradiation, blue data) and $0.95 \pm 0.1 \mathrm{nS}$ (with UV irradiation, red data) for the positive currents and $0.24 \pm 0.02$ $\mathrm{nS}$ (without UV irradiation) and $0.35 \pm 0.05 \mathrm{nS}$ (with UV irradiation) for the negative current. The ion transport conductances present a $100 \%$ increase upon UV irradiation compared to the same experiment in the dark, a value similar to the one previously observed with HPTS assays (Figure 2e). Further comparison of the two sets of perfectly fitted current versus voltage curves indicate that channels of A present an interesting rectification behavior, as confirmed by the different conductance values determined at negative and positive potentials. Such rectification behaviours ${ }^{39}$ are reminiscent with a specific dipolar orientation of the asymmetric superstructures of compound $\mathbf{A}$ within the membrane bilayer (see also CD spectra in Figure S3b).

\section{CONCLUSION}

In summary, we have designed intriguing ion channels constructs based on 18-crown-6 macrocycles conjugated with lightdriven rotary molecular motors. Such channels show selective transport of alkali cations through phospholipid bilayers in the dark. Further, their transport activity strongly increases - up to $400 \%$ - under UV irradiation. To rationalize this effect, one can propose that the actuation dynamics of the molecular motor provides energy to the ion channel in the form of mechanical deformations and vibrations, ${ }^{40}$ or in the form of local heating by energy dissipation. This adds to the surrounding thermal energy and helps crossing the activation barrier necessary to translocate ions between macrocycles along the artificial pore. This first observation of a selective and accelerated cation transport provided by an artificial molecular motor is of importance for the field of molecular machines where such applications are highly expected. The experiments performed in this study strongly suggest that the continuous out-of-equilibrium ${ }^{41}$ rotation of molecular motor $\mathbf{A}$ is responsible for boosting the ion transport activity, which is a fundamentally different functioning principle when compared to molecular switches which can change ion conductivity by adopting different configurations/conformations as a function of their states. These results are also of particular interest for the development of new regulated transport mechanisms making use of responsive artificial channels. This motional activation gives to the channels an increase in performance from the point of view of mass transfer. They can adapt dynamic transient superstructures which are ion selective and permeable. In addition, for the particular case of the patch clamp experiment, this study demonstrates the intriguing coupling between mechanical forces with electrical signaling, a phenomenon at the core of very important pathogenic mechanisms in biology, such as those involved in neurodegenerative diseases. ${ }^{42}$ 


\section{ASSOCIATED CONTENT}

Supporting Information. Synthetic protocols and characterization of organic compounds, corresponding ${ }^{1} \mathrm{H}$ and ${ }^{13} \mathrm{NMR}$ spectra as well as HRMS spectra, protocols for transmembrane transport experiments and planar bilayer lipid clamp assay, additional UV-Vis and DLS experiments. This material is available free of charge via the Internet at http://pubs.acs.org.

\section{AUTHOR INFORMATION}

\section{Corresponding Authors}

* mihail-dumitru.barboiu@umontpellier.fr

* giuseppone@unistra.fr

\section{ACKNOWLEDGMENT}

The authors wish to acknowledge the LabEx CSC at the University of Strasbourg, the Agence Nationale de la Recherche for financial support (ANR-18-CE06-0004-02), and the Chinese Scholarship Council for a fellowship to W.-Z. W., L.-B. H. and S.-P. Z. We are grateful to Dr. Damien Dattler for fruitful discussions on this project.

\section{REFERENCES}

(1) Saier, M. H. Families of Proteins Forming Transmembrane Channels. J. Membr. Biol. 2000, 175 (3), 165-180. https://doi.org/10.1007/s00232001065.

(2) King, L. S.; Kozono, D.; Agre, P. From Structure to Disease: The Evolving Tale of Aquaporin Biology. Nat. Rev. Mol. Cell Biol. 2004, 5 (9), 687-698. https://doi.org/10.1038/nrm1469.

(3) Cukierman, S. Proton Mobilities in Water and in Different Stereoisomers of Covalently Linked Gramicidin A Channels. Biophys. J. 2000, 78 (4), 1825-1834. https://doi.org/10.1016/S0006-3495(00)76732-4.

(4) Watson, M. A.; Cockroft, S. L. Man-Made Molecular Machines: Membrane Bound. Chem. Soc. Rev. 2016, 45 (22), 6118-6129. https://doi.org/10.1039/C5CS00874C.

(5) Watson, M. A.; Cockroft, S. L. An Autonomously Reciprocating Transmembrane Nanoactuator. Angew. Chem. Int. Ed. 2016, 55 (4), 1345-1349. https://doi.org/10.1002/anie.201508845.

(6) Chen, S.; Wang, Y.; Nie, T.; Bao, C.; Wang, C.; Xu, T.; Lin, Q.; Qu, D.-H.; Gong, X.; Yang, Y.; et al. An Artificial Molecular Shuttle Operates in Lipid Bilayers for Ion Transport. J. Am. Chem. Soc. 2018, 140 (51), 1799217998. https://doi.org/10.1021/jacs.8b09580.

(7) Wang, C.; Wang, S.; Yang, H.; Xiang, Y.; Wang, X.; Bao, C.; Zhu, L.; Tian, H.; Qu, D. A Light-Operated Molecular Cable Car for Gated Ion Transport. Angew. Chem. Int. Ed. 2021, 60 (27), 14836-14840. https://doi.org/10.1002/anie.202102838.

(8) Sisson, A. L.; Shah, M. R.; Bhosale, S.; Matile, S. Synthetic Ion Channels and Pores (2004-2005). Chem. Soc. Rev. 2006, 35 (12), 1269-1286. https://doi.org/10.1039/B512423A.

(9) Gokel, G. W.; Leevy, W. M.; Weber, M. E. Crown Ethers: Sensors for Ions and Molecular Scaffolds for Materials and Biological Models. Chem. Rev. 2004, 104 (5), 27232750. https://doi.org/10.1021/cr020080k.

(10) MacKinnon, R. Potassium Channels and the Atomic Basis of Selective Ion Conduction (Nobel Lecture). Angew. Chem. Int. Ed. 2004, 43 (33), 4265-4277. https://doi.org/10.1002/anie.200400662.

(11) Langton, M. J. Engineering of Stimuli-Responsive Lipid-

Bilayer Membranes Using Supramolecular Systems. Nat. Rev. Chem. 2021, 5 (1), 46-61. https://doi.org/10.1038/s41570-020-00233-6.

Banghart, M. R.; Volgraf, M.; Trauner, D. Engineering Light-Gated Ion Channels. Biochemistry 2006, 45 (51), 15129-15141. https://doi.org/10.1021/bi0618058.

Banghart, M. R.; Mourot, A.; Fortin, D. L.; Yao, J. Z.; Kramer, R. H.; Trauner, D. Photochromic Blockers of Voltage-Gated Potassium Channels. Angew. Chem. Int. Ed. 2009, 48 (48), 9097-9101. https://doi.org/10.1002/anie.200904504.

(14) Jog, P. V.; Gin, M. S. A Light-Gated Synthetic Ion Channel. Org. Lett. 2008, 10 (17), 3693-3696. https://doi.org/10.1021/o18013045.

Kerckhoffs, A.; Langton, M. J. Reversible Photo-Control over Transmembrane Anion Transport Using VisibleLight Responsive Supramolecular Carriers. Chem. Sci. 2020, 11 (24), 6325-6331. https://doi.org/10.1039/D0SC02745F.

(16) Yang, R.-Y.; Bao, C.-Y.; Lin, Q.-N.; Zhu, L.-Y. A LightRegulated Synthetic Ion Channel Constructed by an Azobenzene Modified Hydraphile. Chinese Chem. Lett. 2015, $26 \quad$ (7), 851-856. https://doi.org/10.1016/j.cclet.2015.05.010.

Liu, T.; Bao, C.; Wang, H. Lin, Y ; Jia, H. Zhu, L. LightControlled Ion Channels Formed by Amphiphilic Small Molecules Regulate Ion Conduction via Cis-Trans Photoisomerization. Chem. Commun. 2013, 49 (87), 10311-10313. https://doi.org/10.1039/c3cc45618h.

(18) Zhou, Y.; Chen, Y.; Zhu, P.-P.; Si, W.; Hou, J.-L.; Liu, Y. Reversible Photo-Gated Transmembrane Channel Assembled from an Acylhydrazone-Containing Crown Ether Triad. Chem. Commun. 2017, 53 (26), 3681-3684. https://doi.org/10.1039/C7CC01123G.

(19) Zhu, Y.; Fujiwara, M. Installing Dynamic Molecular Photomechanics in Mesopores: A Multifunctional Controlled-Release Nanosystem. Angew. Chem. Int. Ed. 2007, 46 (13), 2241-2244. https://doi.org/10.1002/anie.200604850.

(20) García-López, V.; Chen, F.; Nilewski, L. G.; Duret, G.; Aliyan, A.; Kolomeisky, A. B.; Robinson, J. T.; Wang, G.; Pal, R.; Tour, J. M. Molecular Machines Open Cell Membranes. Nature 2017, 548 (7669), 567-572. https://doi.org/10.1038/nature23657.

(21) Ribovski, L.; Zhou, Q.; Chen, J.; Feringa, B. L.; van Rijn, P.; Zuhorn, I. S. Light-Induced Molecular Rotation Triggers on-Demand Release from Liposomes. Chem. Commun. 2020, $56 \quad$ (62), 8774-8777. https://doi.org/10.1039/D0CC02499F.

(22) van Dijken, D. J.; Chen, J.; Stuart, M. C. A.; Hou, L.; Feringa, B. L. Amphiphilic Molecular Motors for Responsive Aggregation in Water. J. Am. Chem. Soc. 2016, 138 (2), 660-669. https://doi.org/10.1021/jacs.5b11318.

(23) Corra, S.; Curcio, M.; Baroncini, M.; Silvi, S.; Credi, A Photoactivated Artificial Molecular Machines That Can Perform Tasks. Adv. Mater. 2020, 32 (20), 1906064 https://doi.org/10.1002/adma.201906064.

(24) Moulin, E.; Faour, L.; Carmona-Vargas, C. C.; Giuseppone, N. From Molecular Machines to StimuliResponsive Materials. Adv. Mater. 2020, 32 (20), 1906036. https://doi.org/10.1002/adma.201906036.

(25) Feng, Y.; Ovalle, M.; Seale, J. S. W.; Lee, C. K.; Kim, D. J.; Astumian, R. D.; Stoddart, J. F. Molecular Pumps and Motors. J. Am. Chem. Soc. 2021, 143 (15), 5569-5591. https://doi.org/10.1021/jacs.0c13388.

(26) Gilles, A.; Barboiu, M. Highly Selective Artificial K + 
Channels: An Example of Selectivity-Induced Transmembrane Potential. J. Am. Chem. Soc. 2016, 138 (1), 426-432. https://doi.org/10.1021/jacs.5b11743.

(27) Schneider, S.; Licsandru, E.-D. E.-D.; Kocsis, I.; Gilles, A.; Dumitru, F.; Moulin, E.; Tan, J.; Lehn, J.-M. J.-M.; Giuseppone, N.; Barboiu, M. Columnar Self-Assemblies of Triarylamines as Scaffolds for Artificial Biomimetic Channels for Ion and for Water Transport. J. Am. Chem. Soc. 2017, 139 (10), 3721-3727. https://doi.org/10.1021/jacs.6b12094.

(28) Li, Q.; Foy, J. T.; Colard-Itté, J.-R.; Goujon, A.; Dattler, D.; Fuks, G.; Moulin, E.; Giuseppone, N. Gram Scale Synthesis of Functionalized and Optically Pure Feringa's Motors. Tetrahedron 2017, 73 (33), 4874-4882. https://doi.org/http://dx.doi.org/10.1016/j.tet.2017.05.023

(29) Li, Q.; Fuks, G.; Moulin, E.; Maaloum, M.; Rawiso, M.; Kulic, I.; Foy, J. T.; Giuseppone, N. Macroscopic Contraction of a Gel Induced by the Integrated Motion of Light-Driven Molecular Motors. Nat. Nanotechnol. 2015, 10 (1), 161-165. https://doi.org/10.1038/nnano.2014.315.

(30) Sidorov, V.; Kotch, F. W.; Abdrakhmanova, G.; Mizani, R.; Fettinger, J. C.; Davis, J. T. Ion Channel Formation from a Calix[4]Arene Amide That Binds HCl. J. Am. Chem. Soc. 2002, 124 (10), 2267-2278. https://doi.org/10.1021/ja012338e.

(31) Benke, B. P.; Madhavan, N. Active Ion Transporters from Readily Accessible Acyclic Octapeptides Containing 3Aminobenzoic Acid and Alanine. Chem. Commun. 2013, 49 (66), 7340-7342. https://doi.org/10.1039/c3cc44224a.

(32) Alexandratos, S. D.; Stine, C. L. Synthesis of IonSelective Polymer-Supported Crown Ethers: A Review. React. Funct. Polym. 2004, 60, 3-16. https://doi.org/10.1016/j.reactfunctpolym.2004.02.006.

(33) Kado, S.; Takeshima, Y.; Nakahara, Y.; Kimura, K. Potassium-Ion-Selective Sensing Based on Selective Reflection of Cholesteric Liquid Crystal Membranes. J. Incl. Phenom. Macrocycl. Chem. 2012, 72 (1-2), 227232. https://doi.org/10.1007/s10847-011-9970-1.

(34) Yu, H.-R.; Hu, J.-Q.; Lu, X.-H.; Ju, X.-J.; Liu, Z.; Xie, R.; Wang, W.; Chu, L.-Y. Insights into the Effects of 2:1
"Sandwich-Type" Crown-Ether/Metal-Ion Complexes in Responsive Host-Guest Systems. J. Phys. Chem. B 2015, 119 (4), 1696-1705. https://doi.org/10.1021/jp5079423.

(35) Otis, F.; Racine-Berthiaume, C.; Voyer, N. How Far Can a Sodium Ion Travel within a Lipid Bilayer? J. Am. Chem. Soc. 2011, 133 (17), 6481-6483. https://doi.org/10.1021/ja110336s.

(36) Koumura, N.; Geertsema, E. M.; van Gelder, M. B.; Meetsma, A.; Feringa, B. L. Second Generation LightDriven Molecular Motors. Unidirectional Rotation Controlled by a Single Stereogenic Center with NearPerfect Photoequilibria and Acceleration of the Speed of Rotation by Structural Modification. J. Am. Chem. Soc. 2002, 124 (18), 5037-5051. https://doi.org/10.1021/ja012499i.

(37) Chui, J. K. W.; Fyles, T. M. Ionic Conductance of Synthetic Channels: Analysis, Lessons, and Recommendations. Chem. Soc. Rev. 2012, 41 (1), 148175. https://doi.org/10.1039/C1CS15099E.

(38) Cazacu, A.; Tong, C.; van der Lee, A.; Fyles, T. M.; Barboiu, M. Columnar Self-Assembled Ureido Crown Ethers: An Example of Ion-Channel Organization in Lipid Bilayers. J. Am. Chem. Soc. 2006, 128 (29), 9541-9548. https://doi.org/10.1021/ja061861w.

(39) Moorhouse, A. J.; Keramidas, A.; Zaykin, A.; Schofield, P. R.; Barry, P. H. Single Channel Analysis of Conductance and Rectification in Cation-Selective, Mutant Glycine Receptor Channels. J. Gen. Physiol. 2002, 119 (5), 411-425. https://doi.org/10.1085/jgp.20028553.

(40) Chen, J.; Kistemaker, J. C. M.; Robertus, J.; Feringa, B. L. Molecular Stirrers in Action. J. Am. Chem. Soc. 2014, 136 (42), 14924-14932. https://doi.org/10.1021/ja507711h.

(41) Out-of-Equilibrium (Supra)Molecular Systems and Materials; Giuseppone, N., Walther, A., Eds.; John Wiley and Sons, 2021.

(42) Barry, J.; Gu, C. Coupling Mechanical Forces to Electrical Signaling. Neurosci. 2013, 19 (2), 145-159. https://doi.org/10.1177/1073858412456088. 


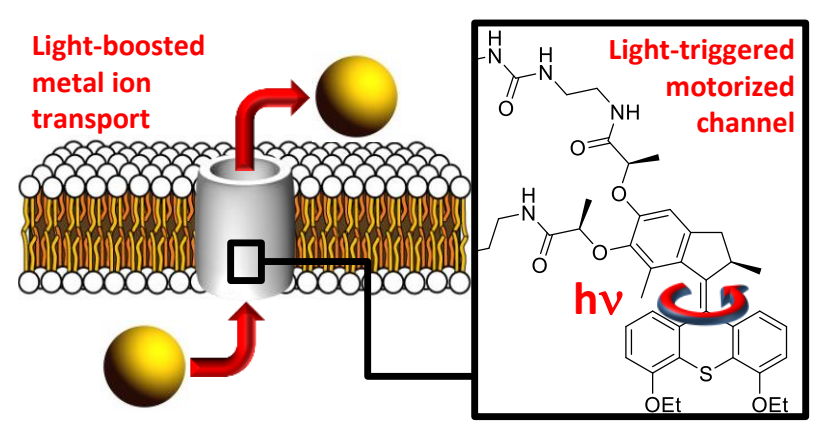

